\title{
SINGULARLY PERTURBED DIRICHLET PROBLEMS WITH SUBQUADRATIC NONLINEARITIES
}

\author{
ALBERT' J. DESANTI
}

\begin{abstract}
Boundary and interior layer theory is provided for a class of singularly perturbed Dirichlet problems with subquadratic nonlinearities in the derivative terms. The results obtained generalize and extend well-known results on the semilinear problem.
\end{abstract}

We aim in this paper to study the asymptotic behavior (as $\varepsilon \rightarrow 0$ ) of solutions of the nonlinear Dirichlet problem

$$
\begin{gathered}
\varepsilon \Delta u=f\left(x, y, u, u_{x}, u_{y}\right) \quad \text { in } \Omega, \\
u(\bar{x}, y, \varepsilon)=g(x, y) \quad \text { on } \Gamma,
\end{gathered}
$$

where $\Delta$ is the Laplacian operator and $\Omega$ is an open set in $R^{2}$ bounded by the smooth closed curve $\Gamma$. The function $f=f(x, y, u, p, q)$ is assumed to be continuous in $x$ and $y$ for $(x, y)$ in $\Omega$ and continuously differentiable in $u, p$, and $q$ for all $u, p$ and $q$. Moreover, it is assumed that the function $f$ is subquadratic in $p$ and $q$ in the sense that the quantity $f(x, y, u, p, q) /\left(p^{2}+q^{2}\right)$ is bounded as $p^{2}+q^{2} \rightarrow \infty$. The function $g$ is assumed to be continuous on the boundary curve $\Gamma$.

The types of asymptotic behavior we consider in this paper are boundary layer behavior and interior shock layer behavior. A solution $u=u(x, y, \varepsilon)$ of problem $(1.1 \mathrm{a}),(1.1 \mathrm{~b})$ is said to exhibit boundary layer behavior if $\lim _{\varepsilon \rightarrow 0} u(x, y, \varepsilon)=$ $U(x, y)$, where $U$ is a classically stable solution of the so-called reduced problem

$$
\begin{gathered}
0=f\left(x, y, U, U_{x}, U_{y}\right) \quad \text { in } \Omega, \\
U(x, y)=g(x, y) \quad \text { on } \Gamma^{\prime} \subset \Gamma,
\end{gathered}
$$

and $U \neq g$ along the boundary segment $\Gamma-\Gamma^{\prime}$. Thus, a solution exhibiting boundary layer behavior remains near a stable reduced solution over most of $\Omega$, but jumps to match the boundary data along $\Gamma$. In contrast to boundary layer behavior, shock layer behavior is characterized by transitions between stable reduced solutions inside $\Omega$. More precisely, a solution $u=u(x, y, \varepsilon)$ of problem (1.1a), (1.1b) exhibits shock layer behavior in the vicinity of a closed curve $C$ properly contained in $\Omega$ if $\lim _{\varepsilon \rightarrow 0} u(x, y, \varepsilon)=U_{1}(x, y)$ for $(x, y)$ inside $C$ and $\lim _{\varepsilon \rightarrow 0} u(x, y, \varepsilon)=U_{2}(x, y)$ for $(x, y)$ outside $C$, where $U_{1}$ and $U_{2}$ are both stable solutions of the reduced equation $(1.2 \mathrm{a})$.

Received by the editors October 6, 1986 and, in revised form, November 6, 1986.

1980 Mathematics Subject Classification (1985 Revision). Primary 35B25, 35J25; Secondary $35 \mathrm{R} 45$.

Key words and phrases. Singular perturbation, Dirichlet problems, subquadratic nonlinearities, differential inequalities. 
Problem (1.1a), (1.1b) has been extensively studied in the case that $f$ is a polynomial function of $u_{x}$ and $u_{y}$. An excellent survey of the linear theory is given in Eckhaus [6]. Semilinear problems, i.e., problems in which $f$ is independent of $u_{x}$ and $u_{y}$, have been studied by Berger and Fraenkel [2], Fife [7], Fife and Greenlee [8], Howes [9], and the author [4]. Quasilinear problems, i.e. problems linear in $u_{x}$ and $u_{y}$, have been studied by Howes $[\mathbf{9 - 1 1}]$ and Kelley [12]. Finally, problems quadratically nonlinear in $u_{x}$ and $u_{y}$ have been studied by Howes [9] and the author [5]. All of these studies make critical use of the polynomial nature of the function $f$. In this paper, we make no polynomial assumptions, but allow $f$ to be much more general in form.

In the next section, we present a boundary layer theory for the subquadratically nonlinear problem (1.1a), (1.1b). In $\S 3$ we concentrate on shock layer behavior in $\Omega$. In both sections, examples are provided in order to illustrate the theory.

2. Boundary layer theory. We begin our study of the boundary layer behavior of solutions of the problem (1.1a), (1.1b) by introducing a coordinate transformation to be used in the vicinity of the boundary $\Gamma$. Let $r(x, y)=\operatorname{dist}((x, y), \Gamma)$ for $(x, y)$ in $\Omega$, and let $z(x, y)$ be the arc length along $\Gamma$ from some reference point to the point on $\Gamma$ closest to the point $(x, y)$. As pointed out in Fife [7], the Jacobian of the transformation $(x, y) \rightarrow(r, z)$ does not vanish in the set $S^{b}=\{(x, y): \operatorname{dist}((x, y), \Gamma) \leq d\}$ for $d$ sufficiently small. The coordinate transformation $(x, y) \rightarrow(r, z)$ is therefore bijective in $S^{b}$.

We shall assume that the boundary curve $\Gamma$ is given by an equation $F(x, y)=0$, where $\nabla F \neq 0$ along $\Gamma$ and $\nabla F$ is an outward normal to $\Gamma$. We note that the curve $r(x, y)=$ constant is a closed curve parallel to the boundary curve $\Gamma$ so that $\nabla r$ is parallel to $\nabla F$ but oriented in the opposite direction.

The following theorem is our principal result on the boundary layer behavior of solutions of the Dirichlet problem (1.1a), (1.1b).

THEOREM 2.1. Suppose that in addition to the conditions described above, the following conditions hold:

(a) the reduced problem (1.2a), (1.2b) has a solution $U=U(x, y)$ which is twice continuously differentiable for $(x, y)$ in $\Omega$; $\Omega$;

(b) $f_{u}\left(x, y, U(x, y), U_{x}(x, y), U_{y}(x, y)\right) \geq K$ for some $K>0$ and for all $(x, y)$ in

$$
(g(x, y)-U(x, y)) \int_{U(x, y)}^{\eta} f\left(x, y, u, U_{x}(x, y), U_{y}(x, y)\right) d u>0
$$

for all $\eta$ such that $U(x, y)<\eta \leq g(x, y)$ or $g(x, y) \leq \eta<U(x, y)$ and for all $(x, y)$ on $\Gamma-\Gamma^{\prime}$

(d) $\left(f_{p}(x, y, u, p, q), f_{q}(x, y, u, p, q)\right) \cdot \nabla F(x, y)>0$ for $(x, y)$ on $\Gamma-\Gamma^{\prime}$, for all $u$ such that $U(x, y) \leq u \leq g(x, y)$ or $g(x, y) \leq u \leq U(x, y)$, and for all $p$ and $q$.

Then, for $\varepsilon$ sufficiently small the problem (1.1a), (1.1b) has a solution $u=$ $u(x, y, \varepsilon)$ such that $\lim _{\varepsilon \rightarrow 0} u(x, y, \varepsilon)=U(x, y)$ for all $(x, y)$ in $\Omega$.

REMARKS. Condition (b) is a classical stability condition on the solution $U$ of the reduced problem. Conditions (c) and (d) are boundary layer stability conditions. Indeed, condition (c) is a well-known condition for boundary layer stability 
for the semilinear case, i.e., the case in which $f$ is independent of $u_{x}$ and $u_{y}$ (cf. Fife [7]). Condition (d) implies that the characteristics of the reduced problem (1.2a), (1.2b) are strictly outgoing along $\Gamma-\Gamma^{\prime}$. That such characteristic behavior is important for boundary layer stability in the quasilinear and quadratically nonlinear cases has been demonstrated by Howes $[\mathbf{1 0}, \mathbf{1 1}]$ and the author $[\mathbf{5}]$.

Proof. Since $f$ is subquadratic in $u_{x}$ and $u_{y}$, we may use the method of suband supersolutions as described, for example, in Amann [1]. That is, we shall construct smooth functions $\underline{u}=\underline{u}(x, y, \varepsilon)$ and $\bar{u}=\bar{u}(x, y, \varepsilon)$ such that $\underline{u} \leq \bar{u}$ in $\bar{\Omega}$, $\varepsilon \Delta \underline{u} \geq f\left(x, y, \underline{u}, \underline{u}_{x}, \underline{u}_{y}\right)$ in $\Omega, \varepsilon \Delta \bar{u} \leq f\left(x, y, \bar{u}, \bar{u}_{x}, \bar{u}_{y}\right)$ in $\Omega$, and $\underline{u} \leq g \leq \bar{u}$ along $\Gamma$. Under these conditions, there exists a solution $u=u(x, y, \varepsilon)$ of the problem (1.1a), (1.1b) such that $\underline{u}(x, y, \varepsilon) \leq u(x, y, \varepsilon) \leq \bar{u}(x, y, \varepsilon)$.

For ease of exposition, we give the proof only for the case $U(x, y)<f(x, y)$ along $\Gamma-\Gamma^{\prime}$. The proof for the remaining case is similar.

We define $\underline{u}$ and $\bar{u}$ as follows:

$$
\begin{aligned}
& \underline{u}(x, y, \varepsilon)=U(x, y)-\gamma \varepsilon \quad \text { for }(x, y) \text { in } \Omega, \\
& u(x, y, \varepsilon)= \begin{cases}U(x, y)+\gamma \varepsilon & \text { for }(x, y) \text { in } \Omega-S^{b}, \\
U(r, z)+v(r, z, \varepsilon)+\gamma \varepsilon & \text { for }(x, y) \text { in } S^{b},\end{cases}
\end{aligned}
$$

where $\gamma$ is such that $\gamma K>M=\max _{\bar{\Omega}}\{\Delta U(x, y)\}$ and where the function $v$ has the form $v(r, z, \varepsilon)=w(r) \hat{v}(r, z, \varepsilon)$ for $w$ a smooth cut-off function such that $w=1$ for $0 \leq r \leq d / 2, w=0$ for $d>1$, and $0 \leq w \leq 1$ for all $r$. The function $\hat{v}$ satisfies

$$
\begin{gathered}
\varepsilon A(0, z) \hat{v}_{r r}>f\left(0, z, U(0, z)+\hat{v}, U_{x}(0, z), U_{y}(0, z)\right) \quad \text { in } S^{b}, \\
\hat{v}(0, z, \varepsilon)=g(0, z)-U(0, z), \\
\hat{v}>0 \text { and } \hat{v}^{\prime}<0 \quad \text { for all } 0 \leq r \leq d,
\end{gathered}
$$

and

$$
\hat{v} \rightarrow 0 \text { exponentially as } \varepsilon \rightarrow 0 \text { for } r \neq 0,
$$

where $A(r, z)=r_{x}^{2}+r_{y}^{2} \neq 0$ in $S^{b}$ since the Jacobian of the transformation $(x, y) \rightarrow$ $(r, z)$ does not vanish in $S^{b}$. The existence of such a function $\hat{v}$ follows from the semilinear theory of Fife [7] under hypotheses (a), (b), and (c).

We note that since $v \geq 0$, we have $\underline{u}<\bar{u}$ for all $(x, y)$ in $\bar{\Omega}$. Moreover, it follows from the definitions of $\underline{u}$ and $\bar{u}$ that $\underline{u}<g$ and $\bar{u}>g$ along the boundary $\Gamma$. Thus, it remains only to establish that $\underline{u}$ and $\bar{u}$ satisfy the appropriate differential inequalities.

Consider first the function $\underline{u}$. From the Mean Value Theorem we have

$$
\begin{aligned}
\varepsilon \Delta \underline{u}-f\left(x, y, \underline{u}, \underline{u}_{x}, \underline{u}_{y}\right) & =\varepsilon \Delta U-f_{u}\left(x, y, \theta, U_{x}(x, y), U_{y}(x, y)\right)(-\gamma \varepsilon) \\
& >-\varepsilon M+\varepsilon \gamma K>0
\end{aligned}
$$

for $\varepsilon$ sufficiently small, where $\theta$ is such that $-\gamma \varepsilon \leq \theta \leq 0$. Thus, $\underline{u}$ is a subsolution for problem (1.1a), (1.1b) in the sense described above.

In the region $\Omega-S^{b}$, the function $\bar{u}$ has the form $\bar{u}(x, y, \varepsilon)=U(x, y)+\gamma \varepsilon$. Thus, applying the Mean Value Theorem as above, we have

$$
\begin{aligned}
\varepsilon \Delta \bar{u}-f\left(x, y, \bar{u}, \bar{u}_{x}, \bar{u}_{y}\right) & =\varepsilon \Delta U-f_{u}\left(x, y, \theta^{\prime}, U_{x}(x, y), U_{y}(x, y)\right)(\gamma \varepsilon) \\
& \leq \varepsilon M-\varepsilon \gamma K<0
\end{aligned}
$$


for $\varepsilon$ sufficiently small, where $\theta^{\prime}$ is such that $0 \leq \theta^{\prime} \leq \gamma \varepsilon$. The function $\bar{u}$ therefore satisfies the correct differential inequality in the region $\Omega-S^{b}$.

Consider now the behavior of the function $\bar{u}$ in the region $S^{b}$. For $0 \leq r \leq d / 2$ in this region we have

$$
\begin{gathered}
\varepsilon \Delta \bar{u}-f\left(x, y, \bar{u}, \bar{u}_{x}, \bar{u}_{y}\right)=\varepsilon A(0, z) \hat{v}_{r r}-f\left(0, z, U(0, z)+\hat{v}, U_{x}(0, z), U_{y}(0, z)\right) \\
-k\left[\left(f_{p}\left(0, z, U(0, z)+\hat{v}, U_{x}(0, z)+\phi, U_{y}(0, z)+\phi^{\prime}\right),\right.\right. \\
\left.\quad f_{q}\left(0, z, U(0, z)+\hat{v}, U_{x}(0, z)+\phi, U_{y}(0, z)+\phi^{\prime}\right) \cdot \nabla F\right] \hat{v}_{r}+O(1)
\end{gathered}
$$

for $\varepsilon$ and $d$ sufficiently small, where $\phi$ and $\phi^{\prime}$ are such that $0 \leq|\phi| \leq\left|\hat{v}_{x}\right|$ and $0 \leq\left|\phi^{\prime}\right|<\left|\hat{v}_{y}\right|$, and where we have used the fact that $\nabla r=-k \nabla F$ for $k$ a positive function of $(x, y)$. Thus, $\bar{u}$ satisfies the appropriate differential inequality for $0 \leq r \leq d / 2$ in $S^{b}$.

For $r>d / 2$ in $S^{b}$, the function $v$ is a transcendentally small term (T.S.T.), i.e., $v$ is $O\left(\varepsilon^{n}\right)$ for every $n$. Thus, in this portion of $S^{b}$ the function $\bar{u}$ has the form $\bar{u}=U(x, y)+\gamma \varepsilon+$ T.S.T. The proof that $\bar{u}$ is a supersolution for $\varepsilon$ sufficiently small in this portion of $S^{b}$ is therefore analogous to the proof that $\bar{u}$ is supersolution in $\Omega-S^{b}$. For brevity, we omit the details.

We have shown that $\underline{u}$ and $\bar{u}$ are sub- and supersolutions in the sense described at the beginning of the proof. We conclude, therefore, that for $\varepsilon$ sufficiently small there exists a solution $u=u(x, y, \varepsilon)$ of problem $(1.1 \mathrm{a}),(1.1 \mathrm{~b})$ such that $\underline{u}(x, y, \varepsilon) \leq$ $u(x, y, \varepsilon) \leq \bar{u}(x, y, \varepsilon)$. Since

$$
\lim _{\varepsilon \rightarrow 0} \underline{u}(x, y, \varepsilon)=\lim _{\varepsilon \rightarrow 0} \bar{u}(x, y, \varepsilon)=U(x, y),
$$

it must be true that $\lim _{\varepsilon \rightarrow 0} u(x, y, \varepsilon)=U(x, y)$. This completes the proof of Theorem 2.1 .

To illustrate the application of Theorem 2.1, we consider the problem

$$
\begin{gathered}
\varepsilon \Delta u=h\left(x u_{x}+y u_{y}\right)+u \quad \text { in } \Omega, \\
u(x, y, \varepsilon)=1 \quad \text { on } \Gamma,
\end{gathered}
$$

where $\Omega$ is the unit disk in the plane centered at the origin and $\Gamma$ is the unit circle given by $F(x, y)=x^{2}+y^{2}-1=0$. The function $h$ is such that $h(0)=0, h^{\prime}(s)>0$ for all $s$, and $h(s) / s^{2}$ is bounded as $s^{2} \rightarrow \infty$. The reduced equation corresponding to equation (2.1a) is

$$
0=h\left(x U_{x}+y U_{y}\right)+U \quad \text { in } \Omega .
$$

A solution of this equation is $U=0$. We note that this reduced solution does not satisfy the boundary data at any point of $\Gamma$. Thus, boundary layer behavior, if it occurs at all, should occur along the entire boundary.

We proceed to check the conditions of Theorem 2.1. We have $f_{u}\left(x, y, U, U_{x}, U_{y}\right)$ $=1$ for all $(x, y)$, so condition (b) is clearly satisfied. Moreover, $\left(f_{p}, f_{q}\right) \cdot \nabla F=$ $\left(x^{2}+y^{2}\right) h^{\prime}=h^{\prime}>0$ for $(x, y)$ on the boundary $\Gamma$. Finally, we have

$$
\int_{0}^{\eta} f\left(x, y, u, U_{x}, U_{y}\right) d u=\int_{0}^{\eta} u d u=\frac{\eta^{2}}{2}>0
$$

for all $\eta$ such that $0<\eta \leq 1$. Thus, we see that all conditions of Theorem 2.1 are satisfied. We conclude that for $\varepsilon$ sufficiently small the Dirichlet problem (2.1a), (2.1b) has a solution $u=u(x, y, \varepsilon)$ such that $\lim _{\varepsilon \rightarrow 0} u(x, y, \varepsilon)=0$ for $(x, y)$ in $\Omega$. 
3. Shock layer theory. We turn now to a study of the shock layer behavior of the solutions of the Dirichlet problem (1.1a), (1.1b). As mentioned in the Introduction, such behavior is characterized by the rapid transition in $\Omega$ between solutions of the reduced problem (1.2a), (1.2b).

To aid in our shock layer studies, we introduce coordinate transformations analogous to the one used in $\S 2$. First of all, if $C_{1}$ and $C_{2}$ are two closed curves properly contained in $\Omega$, we define $\Omega_{1}$ to be the region inside $C_{1}$ and $\Omega_{2}$ to be the region inside $C_{2}$. For $i=1,2$, let $t_{i}(x, y)= \pm \operatorname{dist}\left((x, y), C_{i}\right)$, where $t_{i}>0$ for $(x, y)$ in $\Omega_{i}$, and $t_{i}<0$ for $(x, y)$ in $\Omega-\Omega_{i}$, and let $s_{i}(x, y)$ be arc length along $C_{i}$ from some reference point to the point on $C_{i}$ closest to $(x, y)$. As is demonstrated in Fife and Greenlee [8], the Jacobian of the transformation $(x, y) \rightarrow\left(t_{i}, s_{i}\right)$ does not vanish in the set $S_{i}=\left\{(x, y): \operatorname{dist}\left((x, y), C_{i}\right) \leq d\right\}$ for some sufficiently small positive number $d$. Thus, the transformation $(x, y) \rightarrow\left(t_{i}, s_{i}\right)$ is bijective in the region $S_{i}$ for $i=1,2$.

We expect that the conditions for shock layer behavior in $\Omega$ should be similar to the conditions for boundary layer behavior along $\Gamma$. Of course, an important aspect of a shock layer theory is the determination, to the extent possible, of the location of the layer in $\Omega$. The following theorem provides sufficient conditions for the problem (1.1a), (1.1b) to have a solution which exhibits shock layer behavior in the region between two particular nested, closed curves properly contained in $\Omega$. For convenience, we assume that boundary layer behavior is absent, however later in this section we show how the theorem may be modified to allow boundary layer behavior in addition to shock layer behavior.

THEOREM 3.1. Suppose that in addition to the conditions on $f, g$, and $\Omega$ described above, the following conditions hold:

(a) There exist two solutions $U_{1}=U_{1}(x, y)$ and $U_{2}=U_{2}(x, y)$ of the reduced problem $(1.2 \mathrm{a})$ and $U_{2}(x, y)=g(x, y)$ along $\Gamma$.

(b) $f_{u}\left(x, y, U_{i}(x, y), U_{i x}(x, y), U_{i y}(x, y)\right) \geq K$ for some $K>0$, for all $(x, y)$ in $\Omega$, and for $i=1,2$.

(c) There exist closed curves $C_{1}$ and $C_{2}$ properly contained in $\Omega$ and given by $J_{1}(x, y)=0$ and $J_{2}(x, y)=0$, respectively, where

$$
J_{i}(x, y)=\int_{U_{1}(x, y)}^{U_{2}(x, y)} f\left(x, y, u, U_{i x}(x, y), U_{i y}(x, y)\right) d u
$$

$\nabla J_{i}$ is an outer normal to the curve $C_{i}, i=1,2$, and $\Omega_{1} \subset \Omega_{2}$.

(d)

$$
\left(U_{1}(x, y)-U_{2}(x, y)\right) \int_{U_{2}(x, y)}^{\eta} f\left(x, y, u, U_{i x}(x, y), U_{i y}(x, y)\right) d u>0
$$

for $(x, y)$ on $C_{i}, i=1,2$, and for all $\eta$ such that $U_{1}(x, y)<\eta<U_{2}(x, y)$ or $U_{2}(x, y)<\eta<U_{1}(x, y)$.

(e) $\left(f_{p}(x, y, u, p, q), f_{q}(x, y, u, p, q)\right) \cdot \nabla J_{1}(x, y)>0$ for $(x, y)$ on $C_{1}$ and

$$
\left(f_{p}(x, y, u, p, q), f_{q}(x, y, u, p, q)\right) \cdot \nabla J_{2}(x, y)<0
$$

for $(x, y)$ on $C_{2}$, for all $u$ such that $U_{1}(x, y) \leq u \leq U_{2}(x, y)$ or $U_{2}(x, y) \leq u \leq$ $U_{1}(x, y)$ and for all $p$ and $q$. 
Then, for $\varepsilon$ sufficiently small there exists a solution $u=u(x, y, \varepsilon)$ of problem (1.1a), (1.1b) such that $\lim _{\varepsilon \rightarrow 0} u(x, y, \varepsilon)=U_{1}(x, y)$ in $\Omega_{1}$ and $\lim _{\varepsilon \rightarrow 0} u(x, y, \varepsilon)=$ $U_{2}(x, y)$ in $\Omega-\Omega_{2}$.

REMARKS. The conditions of Theorem 3.1 imply the existence of a closed curve lying between the curves $C_{1}$ and $C_{2}$ along which shock layer behavior occurs. However, the precise location of this curve is, in general, difficult to determine. We note also that condition (e) implies that the characteristic curves of the reduced equation (1.2a) are outgoing along $C_{1}$ and $C_{2}$ in the sense that these curves point out of the region $\Omega_{1}$ along $C_{1}$ and out of the region $\Omega-\Omega_{2}$ along $C_{2}$. Finally, we note that boundary layer behavior may be easily included in Theorem 3.1. To include such behavior, we need only add to Theorem 3.1 the boundary layer conditions of Theorem 2.1 for $U=U_{2}$.

PROOF. As in the proof of Theorem 2.1, we construct sub- and supersolutions $\underline{u}$ and $\bar{u}$. However, in the present case it is more convenient to construct $\underline{u}$ and $\bar{u}$ to be nonsmooth along the curves $C_{1}$ and $C_{2}$, respectively. Such nonsmooth behavior is permissible provided that $\underline{u}$ and $\bar{u}$ satisfy the relations $D_{l}^{i} \underline{u} \leq D_{r}^{i} \underline{u}$ and $D_{l}^{i} \bar{u} \geq D_{r}^{i} \bar{u}$ along $C_{i}$, where $D_{l}^{i}$ and $D_{r}^{i}$ denote one-sided derivatives with respect to $t_{i}$ along the curve $C_{i}, i=1,2$. A proof of this may be found, for example, in [4].

We shall define $\underline{u}$ and $\bar{u}$ for the case $U_{2}(x, y)<U_{1}(x, y)$ in $\Omega$. Definitions for the remaining case are analogous, as are the details of the proof.

Let $\underline{u}$ and $\bar{u}$ be defined as follows:

$$
\begin{aligned}
& \underline{u}(x, y, \varepsilon)= \begin{cases}U_{2}(x, y)-\gamma \varepsilon & \text { in } \Omega-\Omega_{1} . \\
U_{1}\left(t_{1}, s_{1}\right)+v_{1}\left(t_{1}, s_{1}, \varepsilon\right) & \text { in } \Omega_{1} \cap S_{1} . \\
U_{1}(x, y)-\gamma \varepsilon & \text { in } \Omega_{1}-S_{1},\end{cases} \\
& \bar{u}(x, y, \varepsilon)=\left\{\begin{array}{lr}
U_{2}(x, y)+\gamma \varepsilon & \text { in } \Omega-\left(\Omega_{2} \cup S_{2}\right), \\
U_{2}\left(t_{2}, s_{2}\right)+v_{2}\left(t_{2}, s_{2}, \varepsilon\right)+\gamma \varepsilon & \text { in }\left(\Omega-\Omega_{2}\right) \cap S_{2}, \\
U_{1}(x, y)+\gamma \varepsilon & \text { in } \Omega_{2},
\end{array}\right.
\end{aligned}
$$

where $\gamma$ is such that $\gamma K>\max _{\bar{\Omega}}\left\{\Delta U_{1}, \Delta U_{2}\right\}$ and $v_{1}$ and $v_{2}$ are of the form $v_{i}\left(t_{i}, s_{i}, \varepsilon\right)=w_{i}\left(t_{i}\right) \hat{v}_{i}\left(t_{i}, s_{i}, \varepsilon\right), i=1,2$, where $w_{i}$ is a smooth cut-off function defined in $S_{i}$ such that $w_{i}=1$ for $\left|t_{i}\right| \leq d / 2, w_{i}=0$ for $\left|t_{i}\right|>d$, and $0 \leq w_{i} \leq 1$ for all $t_{i}$. The functions $\hat{v}_{1}$ and $\hat{v}_{2}$ satisfy

$$
\begin{gathered}
\varepsilon A\left(0, s_{1}\right) \hat{v}_{1 t_{1} t_{1}}>f\left(0, s_{1}, U_{1}\left(0, s_{1}\right)+\hat{v}_{1}, U_{1 x}\left(0, s_{1}\right), U_{1 y}\left(0, s_{1}\right)\right), \\
\hat{v}_{1}\left(0, s_{1}, \varepsilon\right)=U_{2}\left(0, s_{1}\right)-U_{1}\left(0, s_{1}\right), \\
\hat{v}_{1}<0, \quad \hat{v}_{1 t_{1}}>0 \quad \text { in } S_{1}, \\
\hat{v}_{1} \rightarrow 0 \text { exponentially as } \varepsilon \rightarrow 0 \text { for } t_{1}>0 \\
\varepsilon A\left(0, s_{2}\right) \hat{v}_{2 t_{2} t_{2}}<f\left(0, s_{2}, U_{2}\left(0, s_{2}\right)+\hat{v}_{2}, U_{2 x}\left(0, s_{2}\right), U_{2 y}\left(0, s_{2}\right)\right), \\
\hat{v}_{2}\left(0, s_{2}, \varepsilon\right)=U_{1}\left(0, s_{2}\right)-U_{2}\left(0, s_{2}\right), \\
\hat{v}_{2}>0, \quad \hat{v}_{2 t_{2}}>0 \quad \text { in } S_{2}, \\
\hat{v}_{2} \rightarrow 0 \text { exponentially as } \varepsilon \rightarrow 0 \text { for } t_{2}<0,
\end{gathered}
$$

where $A\left(t_{i}, s_{i}\right)=t_{i x}^{2}+t_{i y}^{2} \neq 0$ for all $\left(t_{i}, s_{i}\right)$ in $S_{i}, i=1,2$, since the Jacobian of the transformation $(x, y) \rightarrow\left(t_{i}, s_{i}\right)$ does not vanish in $S_{i}$. The existence of such 
functions $\hat{v}_{1}$ and $\hat{v}_{2}$ follows from hypotheses (a), (b), (c), and (d) above and Lemma 2.1 of Fife [7].

From the construction of $\underline{u}$ and $\bar{u}$, it is clear that $\underline{u}<\bar{u}$ in $\bar{\Omega}$, that $\underline{u}<g$ and $\bar{u}>g$ along the boundary $\Gamma$, and that $\underline{u}$ and $\bar{u}$ are not differentiable along the curves $C_{1}$ and $C_{2}$, respectively. Moreover,

$$
D_{l}^{1} \underline{u}\left(0, s_{1}, \varepsilon\right)=U_{2 t_{1}}\left(0, s_{1}\right)<U_{1 t_{1}}\left(0, s_{1}\right)+\hat{v}_{1 t_{1}}\left(0, s_{1}, \varepsilon\right)=D_{r}^{1} \underline{u}\left(0, s_{1}, \varepsilon\right)
$$

and

$$
D_{l}^{2} \bar{u}\left(0, s_{2}, \varepsilon\right)=U_{2 t_{2}}\left(0, s_{2}, \varepsilon\right)+\hat{v}_{2 t_{2}}\left(0, s_{2}, \varepsilon\right)>U_{1 t_{2}}\left(0, s_{2}, \varepsilon\right)=D_{r}^{2} \bar{u}\left(0, s_{2}, \varepsilon\right)
$$

for $\varepsilon$ sufficiently small since $\hat{v}_{1 t_{1}}>0$ for $t_{1}>0, \hat{v}_{2 t_{2}}>0$ for $t_{2}<0$, and $\hat{v}_{1}$ and $\hat{v}_{2}$ both decay to zero exponentially. Thus, the functions $\underline{u}$ and $\bar{u}$ satisfy the appropriate inequalities along the curves $C_{1}$ and $C_{2}$. It remains, therefore, only to establish that the differential inequalities for $\Delta \underline{u}$ and $\Delta \bar{u}$ hold in $\Omega-C_{1}$ and in $\Omega-C_{2}$, respectively.

In the regions $\Omega-\Omega_{1}, \Omega_{1}-S_{1}, \Omega-\left(\Omega_{2} \cup S_{2}\right)$, and $\Omega_{2}$ the proof that $\underline{u}$ and $\bar{u}$ are sub- and supersolutions, respectively, proceeds exactly as in the proof of Theorem 2.1 for the region $\Omega-S^{b}$. Hence, we omit the details and concentrate on the behavior of $\underline{u}$ and $\bar{u}$ in the regions $\Omega_{1} \cap S_{1}$ and $\left(\Omega-\Omega_{2}\right) \cap S_{2}$, respectively.

Consider first the behavior of $\underline{u}$ in the region $\Omega_{1} \cap S_{1}$. For $0 \leq t_{1} \leq d / 2$ we have $v_{1}=\hat{v}_{1}$. Applying the Mean Value Theorem we have

$$
\begin{aligned}
\varepsilon \Delta u- & f\left(x, y, \underline{u}, \underline{u}_{x}, \underline{u}_{y}\right) \\
= & \varepsilon A\left(0, s_{1}\right) \hat{v}_{1 t_{1} t_{1}}-f\left(0, s_{1}, U_{1}\left(0, s_{1}\right)+\hat{v}_{1}, U_{1 x}\left(0, s_{1}\right), U_{1 y}\left(0, s_{1}\right)\right) \\
& -\left[\left(f_{p}\left(0, s_{1}, U_{1}\left(0, s_{1}\right)+\hat{v}_{1}, \xi_{1}, \xi_{2}\right)\right),\right. \\
& \left.\quad f_{q}\left(0, s_{1}, U_{1}\left(0, s_{1}\right)+\hat{v}_{1}, \xi_{1}, \xi_{2}\right) \cdot \nabla t_{1}\right] \hat{v}_{1 t_{1}}+O(1)>0
\end{aligned}
$$

for $\varepsilon$ and $d$ sufficiently small, where $\xi_{1}$ and $\xi_{2}$ are such that $0 \leq\left|\xi_{1}\right| \leq\left|\hat{v}_{1 x}\right|$, and $0 \leq\left|\xi_{2}\right| \leq\left|\hat{v}_{1 y}\right|$. We note that in establishing the above inequality we have used condition (e), the fact that $\nabla t_{1}$ is parallel to $-\nabla J_{1}$ along $C_{1}$, and the fact that $\hat{v}_{1 t_{1}}>0$.

For $t_{1}>d / 2$ in $S_{1}$, the function $v_{1}$ is a transcendentally small term (T.S.T.). Thus, in this portion of $S_{1}$, the function $\underline{u}$ has the form $\underline{u}(x, y, \varepsilon)=U_{1}(x, y)-\gamma \varepsilon+$ T.S.T. The verification that $\underline{u}$ is a subsolution in this region is thus esentially identical to the verification for the region $\Omega-S_{1}$. As above, we omit the details.

Consider now the behavior of the function $\bar{u}$ in the region $\left(\Omega-\Omega_{2}\right) \cap S_{2}$. For $-d / 2 \leq t_{2} \leq 0$ we have $v_{2}=\hat{v}_{2}$. Expanding via the Mean Value Theorem as above we have

$$
\begin{aligned}
\varepsilon \Delta \bar{u}- & f\left(x, y, \bar{u}, \bar{u}_{x}, \operatorname{baru}_{y}\right) \\
= & \varepsilon A\left(0, s_{2}\right) \hat{v}_{2 t_{2} t_{2}}-f\left(0, s_{2}, U_{2}\left(0, s_{2}\right)+\hat{v}_{2}, U_{2 x}\left(0, s_{2}\right), U_{2 y}\left(0, s_{2}\right)\right) \\
& \quad-\left[\left(f_{p}\left(0, s_{2}, U_{2}\left(0, s_{2}\right)+\hat{v}_{2}, \xi_{1}^{\prime}, \xi_{2}^{\prime}\right),\right.\right. \\
& \left.\left.\quad f_{q}\left(0, s_{2}, U_{2}\left(0, s_{2}\right)+\hat{v}_{2}, \xi_{1}^{\prime}, \xi_{2}^{\prime}\right)\right) \cdot \nabla t_{2}\right] \hat{v}_{2 t_{2}}+O(1) \\
< & 0
\end{aligned}
$$

for $\varepsilon$ and $d$ sufficiently small, where $\xi_{1}^{\prime}$ and $\xi_{2}^{\prime}$ are such that $0 \leq\left|\xi_{1}^{\prime}\right| \leq\left|\hat{v}_{2 x}\right|$ and $0 \leq\left|\xi_{2}^{\prime}\right| \leq\left|\hat{v}_{2 y}\right|$. As above, we have made use of condition (e), the fact that $\nabla t_{2}$ is parallel to $-\nabla J_{2}$, and the fact that $\hat{v}_{2 t_{2}}>0$. 
For $t_{2}<-d / 2$ in $S_{2}$, the function $v_{2}$ is transcendentally small, so the verification that $\bar{u}$ is a supersolution in this portion of $S_{2}$ is analogous to the verification that $\underline{u}$ is a subsolution in $S_{1}$ for $t_{1}>d / 2$. Thus we omit the details.

We have shown that $\underline{u}$ and $\bar{u}$ are sub- and supersolutions, respectively, in all of $\Omega$. It follows, therefore, that for $\varepsilon$ sufficiently small there exists a solution $u=u(x, y, \varepsilon)$ of problem (1.1a), (1.1b) such that $\underline{u}(x, y, \varepsilon) \leq u(x, y, \varepsilon) \leq \bar{u}(x, y, \varepsilon)$. From the construction of $\underline{u}$ and $\bar{u}$, it follows that $\lim _{\varepsilon \rightarrow 0} u(x, y, \varepsilon)=U_{1}(x, y)$ in $\Omega_{1}$ and $\lim _{\varepsilon \rightarrow 0} u(x, y, \varepsilon)=U_{2}(x, y)$ in $\Omega-\Omega_{2}$. This completes the proof of the theorem.

An application of Theorem 3.1 is afforded by the example

$$
\begin{gathered}
\varepsilon \Delta u=\left(2 \rho^{2}-1\right) h\left(x u_{x}+y u_{y}\right)+\left(u^{2}-1\right)(u-\delta(\rho)) \text { in } \Omega, \\
u(x, y, \varepsilon)=0 \quad \text { along } \Gamma,
\end{gathered}
$$

where $\rho^{2}=x^{2}+y^{2}, \Omega, \Gamma$, and $h$ are as in the example of $\S 2$, and $\delta(\rho)=$ $\left(2 \rho^{2}-1\right)\left(3 \rho^{2}-2\right) / 3$. We note that the reduced problem corresponding to problem (3.1a), (3.1b) has the solutions $U_{1}=1$ and $U_{2}=-1$. These reduced solutions are stable since $f_{u}\left(x, y, U_{1}, U_{1 x}, U_{1 y}\right)=2(1+\delta(\rho))>0$ and $f_{u}\left(x, y, U_{2}, U_{2 x}, U_{2 y}\right)=$ $2(1-\delta(\rho))>0$.

Turning to the shock layer integral conditions of Theorem 3.1, we see that

$$
J_{i}(x, y)=\int_{-1}^{1} f\left(x, y, u, U_{i x}, U_{i y}\right) d u=\int_{-1}^{1}\left(u^{2}-1\right)(u-\delta(\rho)) d u=\frac{8}{3} \delta(\rho)
$$

which vanishes for $\rho^{2}=1 / 2$ and $\rho^{2}=2 / 3$. Moreover, for $\rho^{2}=1 / 2,2 / 3$ we have

$$
\int_{-1}^{\eta} f\left(x, y, u, U_{i x}, U_{i y}\right) d u=\int_{-1}^{\eta}\left(u^{2}-1\right) u d u=\frac{\left(\eta^{2}-1\right)^{2}}{4}>0
$$

for all $\eta$ such that $-1<\eta<1$. Thus, the interior layer integral conditions of Theorem 3.1 are satisfied along the closed curves $C_{1}$ and $C_{2}$ given by $\rho^{2}=1 / 2$ and $\rho^{2}=2 / 3$. We nute also that $J_{1}=J_{2}$ in this case, and that $\nabla J_{i}$ is an outer normal vector to the curves $C_{1}$ and $C_{2}$.

Let us turn now to the verification of condition (e) of Theorem 3.1. Along the circle $C_{1}$ we have $\left(f_{p}, f_{q}\right) \cdot \nabla J_{i}=-h^{\prime}<0$ since $h^{\prime}>0$ by assumption. Along the circle $C_{2}$ we have $\left(f_{p}, f_{q}\right) \cdot \nabla J_{i}=h^{\prime}>0$. Thus we see that condition (e) of Theorem 3.1 holds.

Since the reduced solution $U_{2}=-1$ does not satisfy the boundary data anywhere on $\Gamma$, we anticipate the presence of boundary layer behavior. Let us check, therefore, the boundary layer conditions of Theorem 2.1. For $(x, y)$ on the boundary $\Gamma$ we have

$$
\begin{aligned}
\int_{-1}^{\eta} f\left(x, y, u, U_{2 x}, U_{2 y}\right) d u & =\int_{-1}^{\eta}\left(u^{2}-1\right)\left(u-\frac{2}{3}\right) d u \\
& =(\eta+1)^{2}\left(9 \eta^{2}-25 \eta+26\right) / 36>0
\end{aligned}
$$

for all $\eta>-1$ since $9 \eta^{2}-25 \eta+26$ has no real roots. We also have $\left(f_{p}, f_{q}\right) \cdot \nabla F=$ $h^{\prime}>0$ by assumption. Thus all conditions of the boundary layer theorem, Theorem 2.1 , are satisfied.

We have shown that the conditions of Theorems 2.1 and 3.1 hold for the problem (3.1a), (3.1b). We conclude then that this problem has a solution $u=u(x, y, \varepsilon)$ 
for $\varepsilon$ sufficiently small which has the property that $\lim _{\varepsilon \rightarrow 0} u(x, y, \varepsilon)=1$ for $(x, y)$ inside $C_{1}$ and $\lim _{\varepsilon \rightarrow 0} u(x, y, \varepsilon)=-1$ for $(x, y)$ in $\Omega$ but outside of $C_{2}$. That is, the solution $u$ exhibits shock layer behavior between the curves $C_{1}$ and $C_{2}$ and boundary layer behavior along the boundary $\Gamma$.

5. Concluding remarks. We have provided in this paper a theory for the asymptotic behavior of solutions of a class of singularly perturbed Dirichlet problems which have subquadratic nonlinearities in the derivative terms. The form of our results makes clear the fact that our theory is an extension and generalization of the now well-known semilinear elliptic theory (cf. $[\mathbf{2}, \mathbf{4}, \mathbf{7}, \mathbf{8}, \mathbf{9}]$ ). In this sense our theory has a certain naturalness and intuitive appeal.

There are two rather obvious questions left open by our theory. First of all, the characteristic conditions used in the results of $\S \S 2$ and 3 are somewhat restrictive. These conditions appear to be necessary for our proofs to succeed. It is natural to wonder, however, if these strong conditions may be weakened in some way. A second question is specific to shock layer theory. In $\S 3$, we were able to prove that a shock layer must occur between two certain nested, closed curves under appropriate conditions. However, the proof provided no clue as to the precise location of the layer. The determination of the precise location of the shock layer in the domain appears difficult, and certainly is left open by our theory.

\section{REFERENCES}

1. H. Amann, Existence and multiplicity theorems for semi-linear elliptic boundary value problems, Math. Z. 150 (1976), 43-63.

2. M. S. Berger and L. E. Fraenkel, On the asymptotic solution of a nonlinear Dirichlet problem, J. Math. Mech. 19 (1970), 553-585.

3. R. Courant and D. Hilbert, Methods of mathematical physics, Vol. II, Wiley Interscience, New York, 1962.

4. A. DeSanti, Boundary and interior layer behavior of solutions of some singularly perturbed semilinear elliptic boundary value problems, J. Math. Pures Appl. 65 (1986), 227-262.

5. __ Singularly perturbed quadratically nonlinear Dirichlet problems, Trans. Amer. Math. Soc. 298 (1986), 733-746.

6. W. Eckhaus, Asymptotic analysis of singular perturbations, North-Holland, Amsterdam, 1979.

7. P. C. Fife, Semilinear boundary value problems with small parameters, Arch. Rational Mech. Anal. 23 (1966/1967), 26-86.

8. P. C. Fife and W. M. Greenlee, Interior transition layers for elliptic boundary value problems with a small parameter, Russian Math. Surveys 29 (1974), 103-131.

9. F. A. Howes, Singularly perturbed semilinear elliptic boundary value problems, Comm. Partial Differential Equations 4 (1979), 1-39.

10. __ Some singularly perturbed nonlinear problems of elliptic type, Nonlinear Partial Differential Equations in Engineering and Applied Science, edited by R. L. Sternberg, A. J. Kalinowsky, and J. S. Papadakis, Dekker, New York, 1980, pp. 151-166.

11. __ Perturbed elliptic problems with essential nonlinearities, Comm. Partial Differential Equations 8 (1983), 847-874.

12. W. G. Kelley, The Dirichlet problem for singularly perturbed quasilinear elliptic equations, J. Differential Equations 40 (1981), 37-52.

Systems Analysis Branch, Code 3196, Naval Weapons Center, China Lake, CALIFORNIA 93555 